DOI: https://doi.org/10.18485/bells.2016.8.11

UDC: 81 '255

UDC: 371.3::811.111

\author{
Mirjana Daničić \\ University of Belgrade \\ Faculty of Philology \\ Serbia
}

\title{
TRANSFORMING THE TRADITIONAL APPROACH TO TRANSLATION TEACHING STRATEGIES
}

\begin{abstract}
The paper presents a different approach to teaching translation where the teacher is seen as a facilitator of the translation task: the lion's share of the transfer of knowledge is accomplished by the student. This approach has been used for teaching non-literary translation, translation assessment and cultural translation for four years now with the third-year students at the English Department of the University of Belgrade. Such an approach involves a mixture of translationoriented reading comprehension strategies, problem-solving of linguistic, semantic and cultural dilemmas, managing "untranslatability" (i.e. what is being lost in translation), disentangling ambiguities. In theory, this approach leans on Jacques Derrida's deconstructive idea that translation always involves transformation. It is his concept that "the text can cross a border and continue, transformed".
\end{abstract}

Key words: translation, non-literary translation, teaching translation, Jacques Derrida, deconstruction, transformation.

\footnotetext{
E-mail address: mirjanadanicic@gmail.com
} 


\section{Introduction}

This paper presents the strategies which have been used for teaching nonliterary translation, translation assessment and cultural translation for four years now with the third-year students at the English Department of the University of Belgrade. This approach involves a mixture of translationoriented reading comprehension strategies, problem-solving of linguistic, semantic and cultural dilemmas, managing "untranslatability" (i.e. what is being lost in translation), disentangling ambiguities. Non-literary translation, as taught at the English Department, refers to the translation of texts: (a) from a variety of areas: science, culture, environment, politics, medicine, economy, law, etc., and (b) written in different registers: journalese English, scientific and technical English, the language of politics and current affairs, legal and court language, business English, banking English, medical English, computer English etc.

In this world, a poor non-literary translation can lead not only to a minor confusion and misunderstanding but it can be a matter of life and death. Hence the importance of training future translators not only in the command of the two languages (the source language and the target language), but also in specific knowledge areas and professional ethics. Bearing all this in mind, in 2006 the English Department introduced a new course - Non-literary Translation. The course has put into focus the following:

- Acquiring further knowledge of the various language characteristics of different registers in English and their implications for translation (in terms of cultural differences, content, word choice, text function, syntactic patterns, information structure, etc.);

- Dealing with the issue of culture-bound language and its implication for translation;

- Recognizing and developing an awareness of the methodology of translation and terminology characteristic of particular subject fields;

- Developing practical research techniques necessary to find solutions for translation problems in the various subject fields;

- Becoming acquainted with different translation assessment strategies. 
Mirjana Daničić: Transforming the Traditional Approach to Translation Teaching Strategies

This has appeared to be a tough task, both for the teacher and the students who have not been taught similar translation techniques before and have not been familiar with many of the topics they now have to translate (they have to learn about new concepts and ideas) either owing to the gaps in high school education or to their personal uninterestedness. Additional difficulties might have arisen from the fact that this approach is far from the author-centred (traditional) translation model. It starts from the text-centred (structuralist) model, taking a little from the readercentred (cognitive) model, but strongly leaning on Jacques Derrida's deconstructive idea that translation always involves transformation. It is his concept that "the text can cross a border and continue, transformed" (Pym 2010: 109). Hence, for the purpose of this paper, we will call this approach a 'transformational' model. Derrida views the translation process "as transaction and as transfer" (Derrida 2001: 176), not as a word-toword conversion:

The operation that consists of converting, turning (convertere, vertere, transvertere) doesn't have to take a text at its word or to take the word literally. It suffices to transmit the idea, the figure, the force. (Derrida 2001: 180)

In this sense-for-sense model, the presupposition is that the translator is familiar not only with the words, but also with the context which provides the sense. The first step - the proper understanding of the context - is related to careful reading and correct comprehension of the source text. Every attempt to teach the translation skill must aim at training the students to transfer the essence of the message and the meaning of the source text accurately. In addition to the reading comprehension ability, the pre-requisite for a satisfactory translation performance is the background knowledge of the subject. It would not be possible to produce a proper translation unless the translator is well-acquainted with the topic. The knowledge of background information, or lack of it, is largely reflected in the quality of students' translations.

In the second step the students need to solve semantic and cultural difficulties. The students have to be constantly conscious of the relationship between semantics and culture. Almost all of them would, at one point, come across a translation problem that requires the 'cultural' approach in order to transfer the meaning faithfully into the target language. As students already know, two cultures (British and Serbian) can be quite 
different, and the fact that the students may have to translate the text written in any of the English-speaking countries in the world - even further complicates their translating task. Therefore, the students are asked to consider social, as well as semantic, meanings of the words they look up - their linguistic and non-linguistic values. When translating, words should be taken as symbols of the culture. This approach, naturally, coincides with avoiding literalness in translating. The meaning of any word or phrase must be considered in terms of the linguistic situation in which it occurs and in order to translate correctly students should study the actual usage.

\section{Theoretical background}

In his essay "What is a 'Relevant' Translation?", Derrida takes the notion that every original (already) lacks its translation. However, he expresses his preference for the term 'transformation' over the term translation which

is defined as "a regulated transformation of one language by another, of one text by another" (Derrida 2004: 19). Derrida's idea of translation is seemingly ambivalent because he is of the opinion that translation is both impossible and necessary - impossible because of the undeniable lack between effable and ineffable, but necessary in order to make this lack functional. To reconcile these two, Derrida defines a relevant translation as a "translation whose economy, in these two senses, is the best possible, the most appropriating and the most appropriate possible." (Derrida 2001: 179, emphasis M.D.)

His ideas on translation make use of linguistics, literature studies, cultural studies, psychoanalysis. Derrida obviously uses the word translation in a very broad sense, underpinning the cultural, psychoanalytic and social (political, theological etc.) component of the translation process (Derrida 2001: 197). Translation in his mind is inextricably linked with the quest for broader knowledge. Thus, translatability lies beyond language and rests upon the meaning. This notion, in a way, shatters the linguistic imperialism in translation and solidifies the view that translation does not merely forward the legacy of the original - it has its own life, and the designer of that life is the translator himself/herself. In the contemporary translation theory, this overlaps with Jeremy Munday's notion that "the translator is not just an innocent mediator" (Daničić \& Josipović 2015/16: 175) whose "main focus is on producing a coherent text". This approach 
Mirjana Daničić: Transforming the Traditional Approach to Translation Teaching Strategies

to the translation practice underlines that the translator's comprehension of the exact meaning of the source text is a critical point in the translation process. It becomes a prerequisite to any proper and truthful translation and it can explain the translator's choices that could have been made, but were not made.

\section{In the classroom}

Derrida's integration of knowledge in the translation process and Munday's perception of translation as a form of mediation or intervention can provide a simple answer to the question 'What has to remain constant in translation?' It is always the meaning. Nouns, verb processes, connectors can have slight variations, adjectives can have somewhat bigger variations, especially when there is no easy equivalent, but the meaning has to remain the same.

Bearing this in mind, in my classroom, the teacher is seen as a facilitator of the translation task: the lion's share of the knowledge transfer is accomplished by the students because translation is not perceived merely as a transfer process from a foreign language to the mother tongue and vice versa, but as the interaction of the two languages, two cultures, or sometimes even two worlds. This approach to translation teaching relies on the profile of the average third-year student that includes sound linguistic training in English (B2+, C1 - or C1), an interest in a variety of cultural, political, economic, and scientific topics, high reading-comprehension competence, knowledge of the translation techniques, and adequate research skills.

The teacher's first step is to introduce the topic to the students. This is done much in advance - at least two or three weeks ahead. Once the students learn about the topic to be dealt with in translation classes, their first assignment is to get well acquainted with it: they can use electronic or printed resources, personal contact with their family members, friends or acquaintances who are experts in the field, or cross-cultural communication. Secondly, they must become aware of the importance of the correct comprehension of the source text and must be made to realize that incorrect comprehension significantly ruins the quality of the translation. Thus, they are instructed to use all available reading comprehension strategies careful reading, recognizing style and register, underlining unknown words 
and phrases, analyzing, inferencing, self-questioning, detecting translation difficulties, contextualizing lexical items instead of isolating them, making glossaries or translation journals, relating background knowledge. The next big step for students is trying to find solutions for dilemmas and making a draft translation. All the said steps/phases are done in advance - before the actual class takes place. Based on the principles of Derrida's deconstructive approach, the students are supposed to use all their previous linguistic training for the sole purpose of producing a meaningful and truthful translation: "Speaking, teaching, writing [...] - I know that these activities are meaningful in my eyes only in the proof of translation, through an experience that I will never distinguish from experimentation." (Derrida 2001: 175, emphasis M.D.). The classroom work can best be described as a constant making of choices, i.e. Derrida's experimentation as a way of resolving uncertainties and precariousness. The translation of each sentence, paragraph, or translation unit is read aloud by a student, as Newmark suggests "translation is for discussion" (Newmark 1995), and everyone present is allowed to make comments, corrections, suggestions, or improvements, while the role of the teacher is to guide the discussion, be the final judge in tricky situations, clarify new concepts and give extra solutions where and if necessary. In this way, translation teaching becomes a cooperative process. Students are encouraged to take notes and make questions about all solutions provided by other students or the teacher. The process stops in the phase of final checking of coherence and cohesion of the translated text, which is done with joint effort.

\section{Translation assessment criteria}

The students' achievement in this course is checked and marked in the translation exam. The translation task in the exam includes the translation of two 250-word-long texts - one to be translated into English, the other from English. The texts are written in two different registers, they are topical and used to test students' competence to solve various linguistic, comprehension, orthographic, translation problems, all context-based. Students are allotted 120 minutes to complete the translation of the texts.

The assessment criteria are completely in sync with this method of translation teaching. The most serious errors, listed in order of seriousness, include the following: 
Mirjana Daničić: Transforming the Traditional Approach to Translation Teaching Strategies

- Mistranslation into the target language - the meaning of the original text is not conveyed properly in the target language;

- Misunderstanding of the original text - misreading, or misinterpreting the words, or the syntax of the source text;

- Incomplete sentence / passage - a substantially unfinished sentence / passage;

- Addition or omission - something is inserted that is not expressed in the original text, or something essential to the meaning is left out;

- Wrong terminology - in the contexts where words often have very specific meanings, it is essential that we select the most appropriate word among several which have similar (but not identical) meanings;

- Register - the register of the source language should be preserved in the translation;

- Too free a translation - "rewriting", or "improving" the original text is forbidden in translation. The tendency "to clarify" the original meaning should be resisted;

- Word-for-word translation - following the source text word by word often results in awkward and incorrect renditions;

- Indecision - giving more than one option;

- Grammar, syntax, punctuation - the rules and conventions of the target language should be followed;

- Style - if the source text is characterized by a distinctive manner of expression, this should be reflected in the translation.

The penalization of errors is explained to the students in advance - before they take the exam. The students must fully understand why they lose points and what should have been translated differently, as well as why they earn points for certain solutions to translation problems. In the previous years, the passing rate in the exam has varied between 55\% and 75\%.

\section{Conclusion}

Evidently, education and trainings for translators - "the ones who know how to read and write" (Derrida 2001: 174-175), should involve a set of translation-oriented skills: text analysis, reading comprehension strategies, 
different language skills advancement techniques, cultural studies. These skills should be employed to encourage translation-driven thinking in students. During the translation course, students must learn the fact that meaning is not conveyed by words solely. They should come to realize that all their translations skills and techniques must aim at transferring the message from the source to target language while being faithful to the meaning of the source text. Therefore, students' translation competences should be appraised in terms of conveying the meaning and sense of the source text, as well as the coherence of the output which is evaluated through the application of proper lexical, grammatical, semantic, cultural, stylistic, orthographic transfer. Seeing translation as a cross-linguistic, cross-cultural means of communication, we transform the rigid traditional approach to translation teaching favouring Grammar Translation, Direct Method, or Classical Method.

\section{References}

Daničić, M. \& S. Josipović. (2015/16). An interview with professor Jeremy Munday: "The translator is not just an innocent mediator". Beograd: Philologia, 175-179.

Derrida, J. (2001). "What is a 'Relevant' Translation?". Critical Inquiry, winter 2001, Chicago: The University of Chicago Press, 174-200.

Derrida, J. (2004). Positions, trans. by A. Bass. New York: Continuum, 16-33.

Newmark, P. (1995). A Textbook of Translation. Library of Congress Cataloging-in-Publication Data.

Pym, A. (2010). Exploring Translation Theories. London: Routledge. 